\title{
Severe Temporal Hyper-Activated States Caused by Noise in Tinnitus and Hyperacusis with Normal Hearing
}

\author{
Eun Bit Bae ${ }^{1,2,3}$ and Jun Ho Lee ${ }^{1,2}$ \\ ${ }^{1}$ Interdisciplinary Program in Brain-Science, Seoul National University, Seoul, Korea \\ ${ }^{2}$ Department of Otorhinolaryngology-Head and Neck Surgery, Seoul National University College of Medicine, \\ Seoul National University Hospital, Seoul, Korea \\ ${ }^{3}$ Department of Otorhinolaryngology-Head and Neck Surgery, Seoul National University Bundang Hospital, Seongnam, Korea
}

\author{
Received November 29, 2018 \\ Revised January 25, 2019 \\ Accepted March 4, 2019
}

Lots of neuroimaging and animal studies have revealed that tinnitus and hyperacusis share the same patterns in the bottom up central auditory process. The aim was to identify the abnormal central patterns commonly observed in both tinnitus and hyperacusis in humans. We investigated two cases of normal hearing: a tinnitus patient and a hyperacusis patient. We compared the differences between the severe temporal hyper-activated state (STHS), with spikes, fast beta and gamma frequencies after noise exposure, and the mild temporal hyperactivated state (MTHS), in no sound exposed condition. The power of the gamma band in the two cases was increased in both auditory cortices compared to the other brain regions. Our results of human with normal hearing were the first to identify how tinnitus and hyperacusis caused by sound are abnormally active and how they maintain constant pathological states.

J Audiol Otol 2019;23(3):160-166

KEY WORDS: Auditory cortex · Noise · Tinnitus · Hyperacusis · Electroencephalography.

\section{Introduction}

There are two otologic disorders in which symptoms are subjective, and yet, there is no objective method to measure the pathological severity. It is common sense that hearing loss is the cause of most tinnitus cases, and the hearing loss matches the tinnitus pitch [1,2]. Another otologic disorder, hyperacusis, is known to be commonly caused by loud and high frequency sounds generated in outside environments. However, recently tinnitus and hyperacusis were shown to share the same patterns of increasing activity which is generated in the middle of the signal transmitting process along the intrinsic auditory or cochlear nerve to the brain. Based on the commonalities of these two different disorders, tinnitus and hyperacusis each develop sharing the same root in central auditory process [3]. Central gain enhancement is observed easily as

This is an Open Access article distributed under the terms of the Creative Commons Attribution Non-Commercial License (https://creativecommons.org/licenses/by-nc/4.0/) which permits unrestricted non-commercial use, distribution, and reproduction in any medium, provided the original work is properly cited. increased activity by neuroimaging of tinnitus and hyperacusis patients. Usually, both otologic disorders show increased activity in various brain areas including the auditory cortex, and these brain areas were affected by various symptoms and types of the disorder, thus previous researches regarding neuroimaging of tinnitus have been revealed more brain areas related to symptoms than hyperacusis.

Neuroimaging methods have repeatedly revealed differences in neuronal activity in the auditory and non-auditory areas including the basal ganglia, posterior cingulate cortex, thalamus and para-hippocampus [4] between tinnitus patients and healthy adults. The confirmed brain areas that had increased activity were the auditory cortex, anterior cingulate cortex and insula as well as the connectivity between the auditory cortex and fronto-parietal attention network [4].

It is still difficult to determine the order of the onset areas for the commonly used functional connectivity in various types of tinnitus. The resting state of electroencephalography (EEG) can represent the default mode of tinnitus in real-time, and one of the strengths of the quantitative EEG methodology 
is that it can measure neuronal malfunctions based on power and oscillation. Additionally, another strength is that abnormal activity can be explained by the shape of the oscillation. The frequency and morphology of brain waves (spike, trigonal, biphasic, etc.) represents the function and pathological state of the brain region [5].

In this study, we closely examined the EEG data focusing on the temporal abnormal hyperactivity. Considering the noise condition of the tinnitus and the hyperacusis subjects, we compared EEG data between a no sound (NS) exposed state and a noise-induced (NI) or noise exposed temporal hyper-activated state. We also suggested the common point of the mechanism in the central pathway for tinnitus and hyperacusis after noise exposure in humans.

\section{Case Report}

Data were collected from two patients each with a different type of subjective otologic disorder, tinnitus and hyperacusis. We used EEG data, i.e. which was pre-treatment and naïve to transcranial stimulation, which were obtained before starting a treatment of the previously performed clinical trials. These studies were approved by the Institutional Review Board of the Seoul National University Bundang Hospital in January 2018 (IRB No. B-1710-427-005) and in April 2017 (IRB No. B-1612-373-001). One of the trials was for assessing effects of the combination of two types of transcranial stimulation on tinnitus with depressed mood and the other study was for assessing objectively effects of the hyperacusis treatment. We obtained written consents twice after providing the clinical significance, purpose and use of the data. The first consents were obtained when both subjects were enrolled for previous studies. The second consents were obtained to participate in this study.

\section{Case 1}

One of the subjects was a 54-year-old male who has suffered from tinnitus for 4.5 years at that time. He was doing manual labor at a construction site which was a very loud noisy environment. He had slight hearing loss of $30 \mathrm{~dB}$ in the left ear and $20 \mathrm{~dB}$ in the right ear at a high frequency of $8 \mathrm{kHz}$. The mean right and left hearing thresholds in the frequency range from 250 to $8,000 \mathrm{~Hz}$ were $14.3( \pm 4.5)$ and $15.7( \pm 6.7)$ $\mathrm{dB}$, respectively (Supplementary Table 1 in the online-only Data Supplement). He complained that the tinnitus intensity was always loud, usually worsening on work days with a better tinnitus status on his days off.

\section{Case 2}

The other patient was a 26-year-old female who complained regarding her chronic hyperacusis. The onset was during middle school; thus, her hyperacusis symptoms had been ongoing for almost ten years at the time of her clinical. She had physical symptoms of hyperacusis in the ipsilateral right ear. When the middle ear muscles were contracted and an ear spasm developed due to an unexpected loud noise, a rustling sound was heard in her ear [6]. She felt only unpleasant at the moment when there was a physical symptom in her ear, especially on the right side. Her hearing was within normal range in right and left sides. Her mean hearing in the frequency range from $250-8,000 \mathrm{~Hz}$ was $5.7( \pm 3.5)$ and $4.3( \pm 1.9) \mathrm{dB}$ in the right and left ears, respectively (Supplementary Table 2 in the online-only Data Supplement).

\section{Electroencephalography test}

The EEG were taken in both subjects with tinnitus or hyperacusis in an electrical and noise shielded room. Using 31 electrodes, resting state EEGs were recorded. While recording the EEG for 5 minutes, there was no sound stimulus except for the subject with hyperacusis (Case 2). And we analyzed and compared epoch time of the silent states of both subjects, after noise-induced (aNI) condition and in no-sound condition. The EEG data were analyzed and compared before and after the subjects were exposed to the noise.

The speech stimulation was given only to the hyperacusis subject. The external sound was from one of the authors, a 28-year-old female voice, and the measured intensity was around 40 decibels. The speech sound was generated outside the shielding booth. The length of the sentence was 2 to $3 \mathrm{sec}-$ onds. Speech intensity under $20 \mathrm{~dB}$ was measured in a noise shielding booth.

Depending on the severity of the auditory cortex activity, considering Fig. 1, we divided temporal cortex activity into the two states, severe temporal hyper-activated state (STHS) and mild temporal hyper-activated state (MTHS). For STHS, EEG data on the working day of case 1 and aNI of case 2 were used, and for MTHS, EEG data on day-off of case 1 and no sound exposed state of case 2 were used.

Regarding the STHS, the post noise-exposed condition for the subjects was denoted as 'aNI' and the STHS was pointed with a filled arrow (Supplementary Fig. 1 in the online-only Data Supplement). Regarding the MTHS, the noise condition was denoted as 'NS' and MTHS was pointed with an empty arrow (Supplementary Fig. 1 in the online-only Data Supplement).

Two groups were compared through Mann-Whitney U test and four groups comparison was done via Kruskal-Wallis 


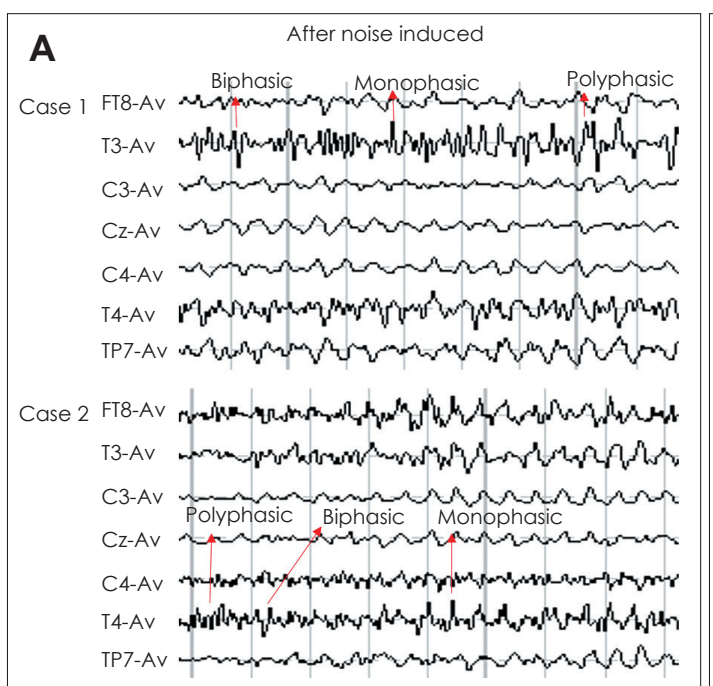

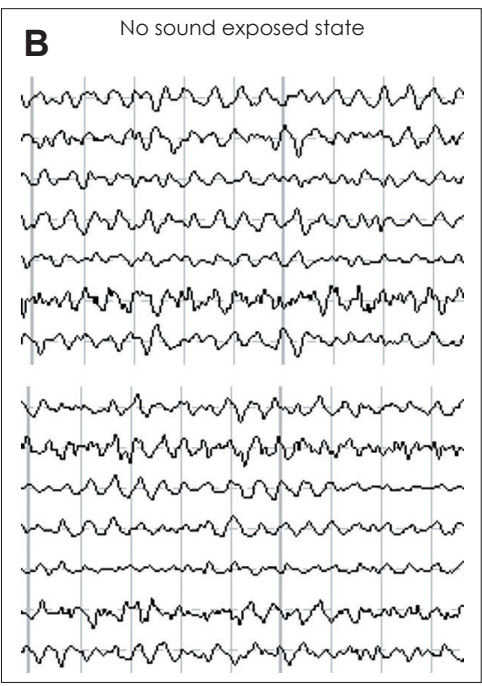

Fig. 1. Enlarged electroencephalogram focused on the STHS and the MTHS. The spikes of the epileptiform were shown in the bilateral auditory cortices after noise exposure. A: STHS after noise exposure. B: MTHS which was observed for the no sound exposed condition. STHS: severe temporal hyper-activated state, MTHS: mild temporal hyperactivated state, $\mathrm{FT}$ : fronto-temporal region, T: temporal, C: central, TP: temporal-parietal, Av: average. test. All statistical analyses were performed via IBM SPSS ver. 23 (IBM Corp., Armonk, NY, USA).

Post-processing of the EEG data and analysis of the topography were described in the supplementary data file.

The wave in the high frequency oscillation had several shapes for the two cases, a spiky and trigonal shape, and sometimes, biphasic shapes appeared after exposure to the noise (Fig. 1). In case 1, EEG was performed in the no sound stimulated state on his working day. Among 31 brain areas, especially a high power oscillation and high frequency were observed in $\mathrm{T} 3$ and $\mathrm{T} 4$, which were located on the primary and secondary auditory cortex, compared to the other brain areas (Supplementary Fig. 1 in the online-only Data Supplement). Severe hyperactivity in the bilateral auditory cortex was maintained throughout the entire time of the EEG test on his working day (Supplementary Fig. 1B in the online-only Data Supplement). Supplementary Fig. 1A (in the online-only Data Supplement) shows a spiky wave on the left side rather than on the right side. Both activities became gradually similar, and at the end of the test, the left and right sides seemed to be the same (Supplementary Fig. 1B in the onlineonly Data Supplement). Two weeks later on his day off, the hyperactivity was still maintained in the bilateral temporal area, but a few minutes after the start of the test, the activity decreased compared to the beginning of the second EEG as well as the first EEG (Supplementary Fig. 1C, D in the onlineonly Data Supplement).

The raw EEG recording for Case 2 showed a slightly faster activity in the right auditory cortex compared to the other regions (Supplementary Fig. 1E in the online-only Data Supplement) before exposure to the sound. A few minutes later in the noise shielding booth, the temporal hyperactivity was decreased (Supplementary Fig. 1F in the online-only Data
Supplement). When the speech sound was heard, the right temporal activity was abnormally excited; thus, the amplitude was higher than before, and pointy and fast oscillations appeared as seen in Supplementary Fig. 1Gb in the online-only Data Supplement. Especially, mainly the right primary and secondary auditory cortex (T4) maintained desynchronized hyperactivities for almost one minute (Supplementary Fig. $1 \mathrm{H}, \mathrm{I}$ in the online-only Data Supplement). Even though there was no sound stimulus in and outside of the booth, the abovementioned abnormal shape of the oscillations lasted for over 50 to 60 seconds (Supplementary Fig. 1I in the online-only Data Supplement). Eighty seconds later after exposure to the speech sound, the right auditory cortex almost seemed to have a similar oscillation pattern as that of the other brain areas.

The fast and high amplitude with an abnormal oscillation shape is represented as a very strong color, dark red, in Fig. 2A. In the tinnitus subject, abnormalgamma signals were strongest and were observed in the only two electrodes, where are the left and right auditory cortex. The gamma/theta power ratios became stronger and much more powerfully colored for the power ratios of (beta3 and gamma)/(delta and theta). In the hyperacusis subject, both the left and right auditory cortices showed a deep red color; additionally, $\mathrm{C} 4$, which is in the post central gyrus, and part of the pre-frontal cortex (F7 and F8) were also dark red.

The MTHS was observed on the day when the subjects were not exposed to a sound or noisy working environment (Fig. 2B). Still, the MTHS of the primary and secondary auditory cortex (Fig. 2, Supplementary Materials in the online-only Data Supplement) in both subjects showed a stronger power at gamma band ratio, gamma/theta ration and the (beta3, gamma)/(delta, theta) ratio more than other brain areas where the 29 electrodes were located. The tinnitus subject had a 
higher activity in the right auditory cortex than the left one, and the hyperacusis subject had hyperactivity in the left instead of the right auditory cortex.

To clinically assess the neuronal activity, we compared the neuronal powers in the seven frequency bands according to each activity state. In the case 1 , the inhibit function of beta and gamma, delta and theta bands outputs were much higher compared with the no sound induced condition. However, the delta and theta power aNI condition was reduced to almost zero and increased sharply at the high frequencies in the auditory cortex from alpha (Fig. 3, Supplementary Table 2 in the online-only Data Supplement) to gamma. And nor-

Fig. 2. The brain activity of each subject was shown in the brain topography. Scale bar (Maximum power thresholds): Gamma (20), Brain oscillation band ratio (300\%). Up side (A and $B$ ): Tinnitus subject (M/54). Down side (A and B): Hyperacusis subject $(F / 26)$. A: Severe temporal hyper-activated state. B: Mild temporal hyper-activated state.
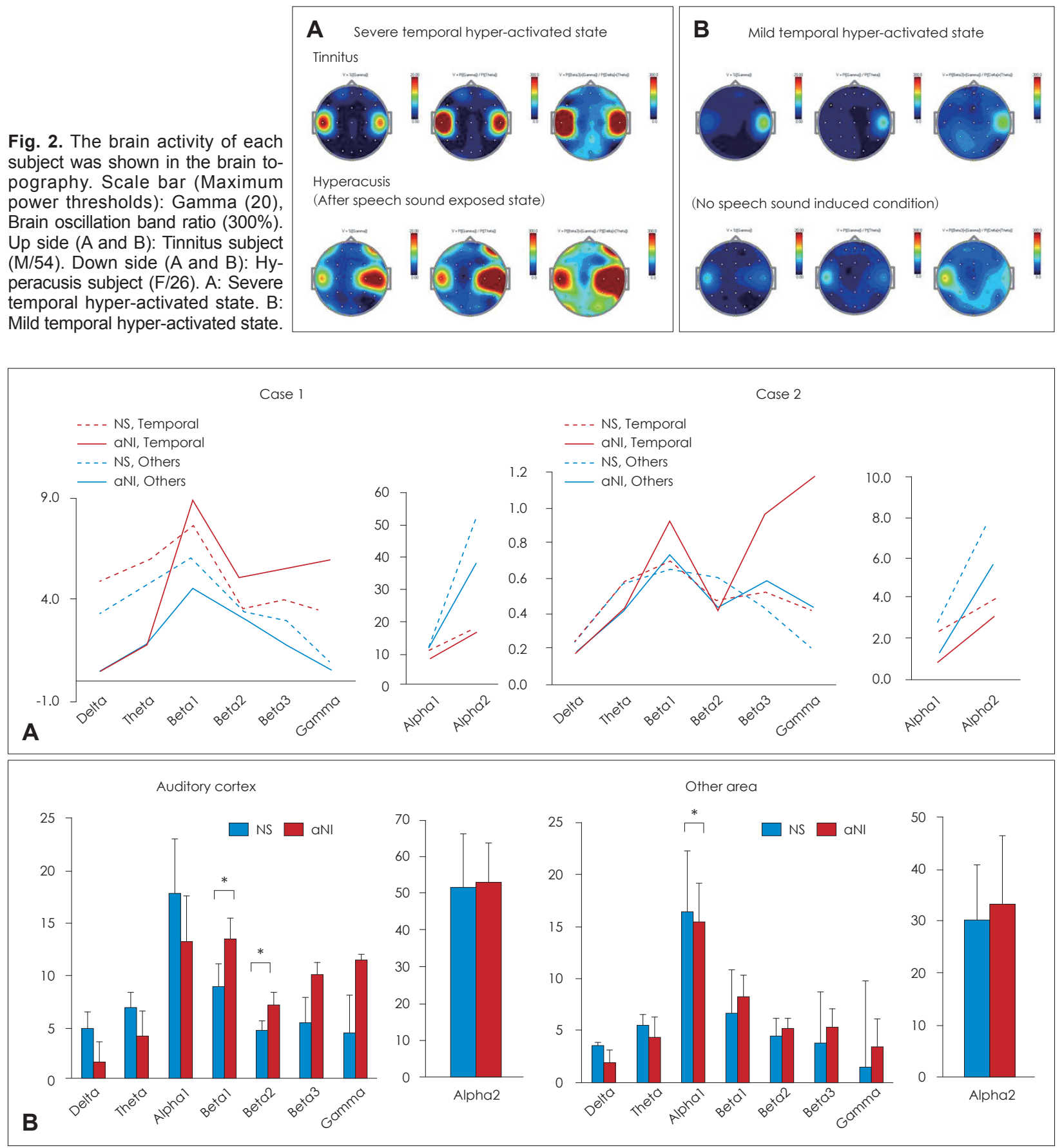

Fig. 3. The comparisons of the neuronal power between the noise conditions were represented in the graph. A: The graph for the comparison among noise conditions and brain areas within each subject. B: The graph for the comparison between no sound exposed state and aNI brain condition. It is represented as the bar graphs on the left side that each state (NS, aNI) of the averaged power density for the bilateral auditory cortices of the two subjects. On the right side of Fig. 4-B, T3, T4, TP7, TP8, and other 27 channels of the other brain areas were averaged each state of the subjects. Error bar: standard deviation. $\mathrm{Y}$ axis $=\mu \mathrm{V}^{2},{ }^{*} p<0.05$. NS: no sound, aNI: after noise-induced. 


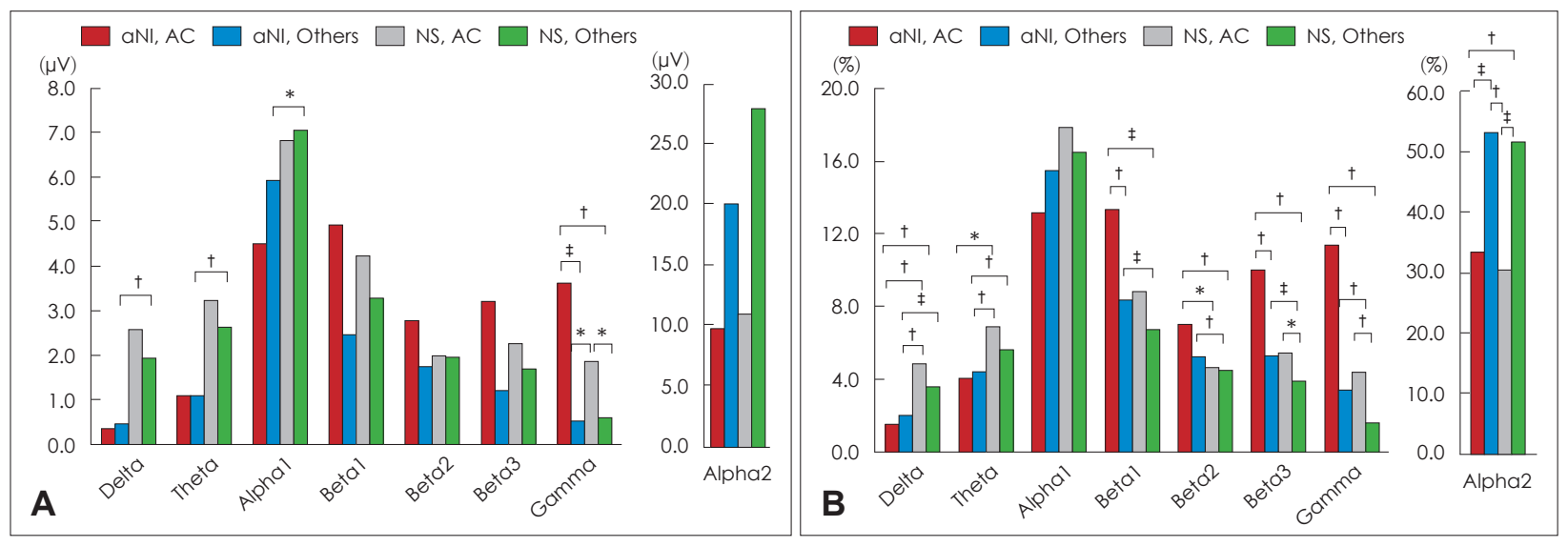

Fig. 4. The results of statistical comparisons of neuronal activity in two noise conditions related auditory or non-auditory cortex. Red colored bar: aNI state in the auditory cortices. Sky-blue colored bar: aNI state in other brain areas. Grey bar: no sound exposed state in the auditory cortices. Green bar: no sound exposed state in other areas. $\mu \mathrm{V}=\mu \mathrm{V}^{2},{ }^{*} p<0.05,{ }^{\dagger} p<0.01,{ }^{\ddagger} p<0.001$. aNI: after noise induced.

mal gamma was commonly observed when normal people do higher sensory processing, listening, or cognition [5].

The case 2 showed a pattern similar to the case 1 , with the lower frequencies slightly higher than the aNI state, and the temporal activity showed a sharp increase at the high frequency bands (Fig. 3). Neuronal power comparisons between aNI state and NS state within the same brain areas, for example, between the auditory cortex of the aNI state and the NS state and between the other brain areas of aNI state and the NS state, were statistically highly significant (Fig. 4, Supplementary Table 3 in the online-only Data Supplement). High differences were observed in the appearance rate of the neuronal activity between the NS state and aNI state, and a much lowered inhibition function of the aNI state was observed, and a much higher activity is represented from the beta 1 to the gamma band in Fig. 4 (statistical results represented in Supplementary Table 4 in the online-only Data Supplement).

\section{Discussion}

Based on animal and cellular studies in vivo and in vitro, the well-verified NI animal and cellular models [2] have helped to establish the concept of central auditory processing disorders caused by noise $[7,8]$. Unlike with micro-electrophysiology such as local field potential (LFP), electrodes of EEG represented broaden brain area than LFP, EEG activity would be synchronous in normal condition [5,9]. In other studies regarding event related potential (ERP) in human, the sound intensity causing temporal desynchronization was louder than $65 \mathrm{~dB}[10,11]$ which is the same level as the noise found in social environments involving daily life [12]. Based on above the studies, our two subjects are in the unique conditions which showed desynchronized and abnormal hyper- activity mainly bilateral auditory cortex after noise exposure in silent condition.

From the results mentioned above, temporal hyper-activated states caused by noise can be classified into two states based on the morphology of the neuronal oscillation. One is the STHS, for which many spikes (Fig. 1) were observed in the auditory cortex after noise exposure, and the other is MTHS which was observed for no exposure to noise on that day. The MTHS did not show spikes (Fig. 1, Supplementary Fig. 1D in the online-only Data Supplement); however, fast beta and gamma frequencies were observed with a more synchronized wave on the auditory cortex (Supplementary Fig. $1 \mathrm{~J}$ in the online-only Data Supplement).

In the results of brain oscillation of the case 2, we could guess the brain states of the hyperacusis subject according to the noise environments. Her resting state of the brain activity in a residential environment may be repeated the $\mathrm{E}, \mathrm{F}$ and $\mathrm{J}$ conditions. However, on her usual working hours in the school, which is her working environment, the brain activity may be maintained G-H-I-(E)-G-H-I-(E) $\cdots$ while exposing to a noise even though it was a small speech sound below 20 dB. In case 1, the EEG which was done on the working day, showed the STHS for the entire 5-minute epoch, and there was no MTHS observed ( $0 \%$ ). However, for the day-off, the EEG showed the MTHS for $30 \%$ of the 300 -second epoch of the EEG recording. The main function of these hyper-activated areas (bilateral auditory cortex), is to recognize the first stage of hearing, sound recognition, and mainly the sounds generated from the outside.

These results suggest that sound can cause an abnormal activity, especially in the primary and secondary auditory cortex, and can develop into chronic tinnitus or hyperacusis if the severe hyper-activated states of the auditory cortex prolonged in a silent environment after noise exposure. The 
reason that the symptoms become to chronic or severe is probably because the hyperacusis subject does not recognize the symptoms when exposed to the sound which is below her uncomfortable level (UCL, Supplementary Table 3 in the online-only Data Supplement).

The subject with hyperacusis showed a slightly elevated activity of the auditory cortex for several minutes in a silent state where no sound was heard in the shielded booth. The case 1 , in which tinnitus was induced by noise, was longer in terms of the duration of the symptoms, from a few hours to a few days, and had a high frequency of severe hyperactivity. The bilateral primary and secondary auditory cortices were abnormally activated from 1 minute (case 2) to several hours or days (case 1) due to an auditory event or a sound stimulus. Continuous exposure sensitizes the neurons of the auditory cortex, and that makes abnormally hyper-activated, resulting in much lower hearing thresholds. This speech sound, smaller than the UCL, resulted in a strong and hysteric response (spiky, trigonal and bi-phasic shape) in the auditory neuron and a desynchronized pattern especially in the primary and secondary auditory cortex for a longer time (Unlike the brain wave, the patient's attitude was remarkably calm, and she did not feel any discomfort when she exposed to speech sounds and did not recognize the difference from the silent condition).

According to previous studies, the noise level in daily life is usually a mean 55-70 $\mathrm{dB}[12]$, and the result of this study showed that temporal desynchronization was observed at an intensity higher than $65 \mathrm{~dB}[8,10,11]$. Compared with previous results, we found that it is different from the general situation of healthy adults and may be different from otologic disorders developed due to other causes. In the case of tinnitus with hearing loss, tinnitus occurred and worsened in the process for intrinsic central auditory processing, and abnormal gain enhancement was observed in various brain regions [3]. In that respect, this research is the first to report the observation of abnormal activity in the beginning of the central auditory process after noise-exposed and no-sound condition in the professions who were long-term exposed to the occupational noise environments.

Based on the above discussions, this case report is further distinguished from the previous studies as follows. When compared based on the subject's hearing, the pathophysiological symptoms are different from other tinnitus and hyperacusis. In the case 1, hearing was much better than in the $50 \mathrm{~s}$ working in the occupational environments similar to that of the subject, i.e. a very loud noise construction site, and even hearing was similar or within same range to normal hearing control $[13,14]$. And in the case of 2 , she is distinguished from general hyperacusis patients in that the abnormal gamma ac- tivity was occurred around the $20 \mathrm{~dB}$ of speech sound, which is lower intensity of her UCL $[8,10,11]$. In this regard, these subjects are assumed special cases that can be distinguished from patho-physiologically to general tinnitus and hyperacusis.

Additionally, as a concept of the central gain enhancement especially in the auditory cortex, recently, several studies have confirmed the same pattern in the pathogenesis of tinnitus and hyperacusis in vivo and in vitro [7]. However, to the best our knowledge, in human, there have been no study was reported that have centrally confirmed a common feature of these two diseases in the developmental process. This is the first time that the affected brain region in the middle of the central auditory process was identified by our EEG results. Furthermore, this study provides basic results that can be applied to understanding the development of two diseases from the abnormal state of two different otologic disorders. In this study, we confirmed that the auditory cortex can be functionally sensitized even with a small sound intensity below the UCL.

For this reason, we cautiously suggest that a therapy using sounds would be carefully considered for NI tinnitus and hyperacusis in normal hearing, because there is a concern that the otologic symptoms may worsen in the initial stage of therapy, even if use sound below UCL (considering above the results, $20 \mathrm{~dB}$ of the speech stimulus for hyperacusis subject was measured in the shielded room). And also we carefully consider that further research would be performed to assess whether sound therapy contributes to overcoming symptoms of chronic NI tinnitus and hyperacusis in normal hearing.

\section{Limitation and further works}

In addition, this study is a retrospective study mainly analyzing the brain oscillations of the two subjects whose otologic disorders were caused by noise exposure. Therefore, it is difficult to know how the EEG response at the time of sound exposure is different from that of the same disease or normal people; thus, this part should be confirmed by a research on a large number of subjects using ERP in another study.

Based on the bottom of Fig. 2 for hyperacusis, we found that the abnormal hyper-activated oscillation was started from the central region of the auditory cortex and moved to the peripheral auditory cortex almost simultaneously. This is one of the results that support the strong connectivity between the primary and secondary auditory cortex and primary somatosensory cortex or motor cortex. With a further work, we might suggest that this process could consequently generate malfunctions in other non-auditory brain areas that were previously mentioned [4] which would reflect tinnitus or hyperacusis development. An additional ongoing study will be 
performed to identify differences of brain activity among NI subjects and other causes induced tinnitus and hyperacusis patients with normal hearing.

\section{Supplementary Materials}

The online-only Data Supplement is available with this article at https://doi.org/10.7874/jao.2018.00514.

\section{Acknowledgments}

We thank to JK and JS for actively involving in the clinical trials. We appreciate professors at the Department of Otorhinolaryngology (OL), Seoul National University Bundang Hospital (SNUBH) for providing us with a place to perform clinical trials. We are deeply grateful to the audiologists at the same department of the Seoul National University Bundang Hospital for measuring hearing thresholds and uncomfortable level of the subjects.

\section{Conflicts of interest}

The authors have no financial conflicts of interest.

\section{Author Contributions}

Conceptualization: Eun Bit Bae. Data curation: Eun Bit Bae. Formal analysis: Eun Bit Bae. Investment: Eun Bit Bae. Methodology: Eun Bit Bae. Resources: Eun Bit Bae. Software: Eun Bit Bae. Supervision: Jun Ho Lee. Validation: Eun Bit Bae. Visualization: Eun Bit Bae. Writing — original drift: Eun Bit Bae. Writing — review \& editing: Eun Bit Bae.

\section{ORCID iDs}

Jun Ho Lee https://orcid.org/0000-0002-5519-3263

Eun Bit Bae https://orcid.org/0000-0002-6148-9909

\section{REFERENCES}

1) Meikle MB, Henry JA, Griest SE, Stewart BJ, Abrams HB, McArdle $\mathrm{R}$, et al. The tinnitus functional index: development of a new clinical measure for chronic, intrusive tinnitus. Ear Hear 2012;33:153-76.

2) Kaltenbach JA, McCaslin DL. Increases in spontaneous activity in the dorsal cochlear nucleus following exposure to high intensity sound: a possible neural correlate of tinnitus. Audit Neurosci 1996;3:57-78.

3) Auerbach BD, Rodrigues PV, Salvi RJ. Central gain control in tinnitus and hyperacusis. Front Neurol 2014;5:206.

4) Shore SE, Roberts LE, Langguth B. Maladaptive plasticity in tinnitus--triggers, mechanisms and treatment. Nat Rev Neurol 2016;12: 150-60.

5) Ebersole JS, Husain AM, Nordli DR. Current practice of clinical electroencephalography. 4th ed. Philadelphia: Lippincott Williams \& Wilkins;2014. p.1-664.

6) Virtanen H. Objective tubal tinnitus: a report of two cases. J laryngol Otol 1983;97:857-62.

7) Hickox AE, Liberman MC. Is noise-induced cochlear neuropathy key to the generation of hyperacusis or tinnitus? J Neurophysiol 2014; 111:552-64.

8) Nalivaiko E, Bondarenko E, Lidström A, Barry RJ. Respiratory component of the orienting reflex: a novel sensitive index of sensoryinduced arousal in rats. Front Physiol 2011;2:114.

9) Eggermont JJ, Tass PA. Maladaptive neural synchrony in tinnitus: origin and restoration. Front Neurol 2015;6:29.

10) Abrams DA, Nicol T, Zecker S, Kraus N. Right-hemisphere auditory cortex is dominant for coding syllable patterns in speech. J Neurosci 2008;28:3958-65.

11) Schadow J, Lenz D, Thaerig S, Busch NA, Fründ I, Herrmann CS. Stimulus intensity affects early sensory processing: sound intensity modulates auditory evoked gamma-band activity in human EEG. Int J Psychophysiol 2007;65:152-61.

12) Ryu IC, Kim HK, Oh KR, Kim GB, Yeo IH, Eom SW. Research on noise levels by frequency analysis in the Seoul Metropolitan Government. Report of Seoul Research of Institute of Public Health and Environment 2011;47:168-82.

13) Lindblad AC, Rosenhall U, Olofsson Å, Hagerman B. Tinnitus and other auditory problems - occupational noise exposure below risk limits may cause inner ear dysfunction. PLoS One 2014;9:e97377.

14) Leensen MC, van Duivenbooden JC, Dreschler WA. A retrospective analysis of noise-induced hearing loss in the Dutch construction industry. Int Arch Occup Environ Health 2011;84:577-90. 